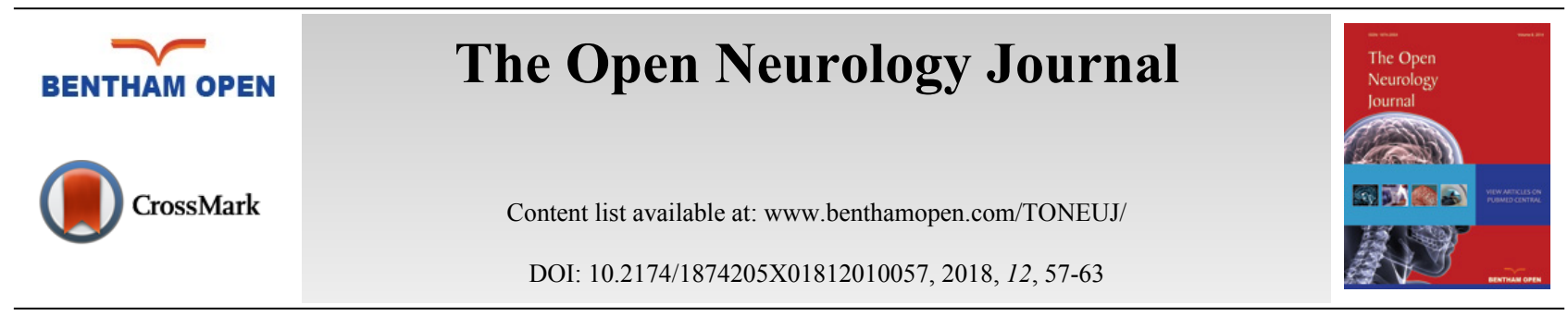

RESEARCH ARTICLE

\title{
The Complication for Ventricular Shunt Based on Different Etiologies: A Prospective Study in Tehran, Iran
}

\author{
Mohammad Vafaee Shahi ${ }^{1}$, Samileh Noorbakhsh ${ }^{2, *}$, Sarvenaz Ashouri ${ }^{3}$, Leila Tahernia ${ }^{4}$ and \\ Maryam Raghami Derakhshani ${ }^{1}$ \\ ${ }^{I}$ Departement of Pediatric, Iran University of Medical Sciences, Tehran, Iran \\ ${ }^{2}$ Departement of Pediatric Infectious Diseases \& Research Center, Iran University of Medical Sciences, Tehran, Iran \\ ${ }^{3}$ ENT and Head and Neck Research Center and Department, Iran University of Medical Sciences, Tehran, Iran \\ ${ }^{4}$ Departement of Pediatric, Tehran University of Medical Sciences, Tehran, Iran
}

Received: July 27, 2018

Revised: August 15, 2018

Accepted: September 30, 2018

\section{Abstract:}

\section{Objectives:}

The purpose of this study was to evaluate and compare complications caused by VP shunt (based on imaging changes, clinical and laboratory findings) in children with congenital (intrauterine) infections, referred to as TORCH, in contrast to other causes of Shunt insertion in children.

\section{Materials and Methods:}

In this cross-sectional, observational and descriptive study, 68 hospitalized patients in Rasool-Akram Hospital were selected using convenience method during 2 years from 2013 to August, 2016. Clinical examination and organ involvement were determined. Blood sampling, serological and complementary tests were performed to determine the type of infection, cause and duration of shunt insertion, mechanical and infectious complications (meningitis and peritonitis), the course of the disease and finally, mortality and morbidity. All data were collected in questionnaires. Statistical analysis was performed using SPSS-24 version software. The comparison was carried out between two groups of infectious and noninfectious causes. P-value less than 5.5 was considered significant.

\section{Results:}

From total 68 children with VP shunt, 13 patients died (including 8 males and 5 females with a mean age of $69.41 \pm 81.57$ monthsold). The main cause of shunt insertion was infection in 4 patients and tumor, cerebral hemorrhage and myelomeningocele in 9 other children. Shunt-induced meningitis and peritonitis were detected in $92 \%$ and $7.7 \%$ respectively. The leading causes of shunt insertion In remaining 47 live children (including 27 males and 19 females with a mean age of $63+83$ months) were infectious in $28 \%$ and noninfectious in the rest of cases. The shunt mechanical complications were reported in $8.7 \%$ (4 patients). Fever was detected in $33 \%$ of patients. Positive blood culture and cerebrospinal fluid were reported in $6.8 \%$ and $8.5 \%$ of patients in that order, but meningitis was detected in $26 \%$ (12 cases) and peritonitis in 10.9\% (5 cases). Although, there were no significant differences between two groups in terms of mechanical complications such as shunt obstruction, cerebral hemorrhage ; but, a considerable difference was reported in death rate due to shunt insertion between two groups with infectious and noninfectious origin. The risk of shunt-induced meningitis was clearly higher in group with infectious origin. (P-value $=0.05)$. But peritonitis risk was not different.

\section{Conclusion:}

VP shunt complications were observed in one-quarter of children, but mechanical complications of VP shunt were uncommon ( $8 \%$ in live patients). VP shunt mortality was relatively high (13 patients from total 68), and occurred more often in patients with non-

\footnotetext{
* Address correspondence to this author at the Departement of Pediatric Infectious Diseases \& Research center, Iran University of Medical Sciences, $4^{\text {th }}$ floor Rasul Akram Hospital, Niayesh Street, Satarkhan Avenue, Tehran, Iran; Tel: 098-21-66525328; E-mail: samileh_noorbakhsh@yahoo.com
} 
infectious causes of VP shunt, mainly due to meningitis. Shunt meningitis was very common In patients with infectious origin. Shunt infection in patients (meningitis, bacteremia and peritonitis) was common and could increase mortality if not controlled. Prescribing antibiotics is not recommended without initial diagnostic measures (such as spinal fluid puncture, ascites, and proper imaging) because it will result in incomplete and improper treatments.

Keywords: Complications, Ventriculoperitoneal Shunt, Children, CSF, Rubella infection, Shunt complications.

\section{INTRODUCTION}

Hydrocephalus refers to the accumulation of additional CSF in the brain. It has two main types: communicative or non-obstructive and obstructive type. In communicative type, there is no obstruction in the CSF flow pathway, but fluid absorption is low by subarachnoid cavity which results in cerebrospinal fluid accumulation in brain spaces. Treatment is shunt insertion: ventriculoatrial shunt, ventriculoperitoneal shunt and other therapeutic methods such as Ventriculostomy. Treatment of choice In hydrocephalus is Ventriculoperitoneal shunt insertion and the use of Ventriculoatrial Shunt (VA) is the second choice. This method can not be used in the presence of bilateral internal jugular vein stenosis or thrombosis. In Ventriculoatrial shunt, one side is placed in the cerebral ventricle and the other side is inserted into the right atrial. In ventriculoperitoneal shunt, one side is located in the cerebral ventricle and the other side into the peritoneal cavity.

Hydrocephalus is treated by redirecting CSF flow from inside of the brain into other spaces that can absorb this fluid. In most cases, shunts consist of three parts that are serially connected to each other: proximal catheter, one-way valve and distal catheter. The most common site to place a proximal catheter is one of the lateral ventricles. The oneway valve also connects proximal catheter to distal catheter, which is usually placed in one of the body cavities. Peritoneal cavity is preferable in this regard [1]. According to published statistics, about 127,000 CSF shunts are annually inserted in the United States [2]. This rate is increasing every year. Although the treatment of children with hydrocephalus is possible by placing a shunt, failure rate is estimated around $40 \%$ in the first year of placement [3]. Shunt complications can be classified into three general categories: mechanical defects, functional defects, and infections. Mechanical defects are associated with either incomplete shunt performance or due to improper shunt placement.

These complications can affect proximal or distal shunt sections. Functional failures point to complications that are created secondary to increased or decreased CSF flow $[4,5]$.

In fact, excessive CSF flow can cause a collapse in the ventricular system and swelling in other parts of the brain. The incidence of shunt infection is between $8-12 \%$ which often occurs during the first six months of shunt insertion [6, 8]

Shunt infection usually results from pollution caused by normal skin flora during surgery, but sometimes it occurs months after surgery, which may be related to infection in a distant place [such as urinary tract infection]. Other potential causes include shunt injury or retrograde infection in the distal catheter [9-11]. Shunt is inserted frequently in Iranian children $[12,13]$. Shunt complications specially shunt-induced infection and resulting bacteria have been documented in different internal and external sources [14, 15]. In our country, like other countries, congenital (intrauterine) infections (which are referred to as Torch) are manifested and diagnosed by a variety of clinical signs [16, 19]. One of the most important clinical manifestations of intrauterine infections, especially in the first months of life, is hydrocephalus with various degrees. It is considered to control intrauterine infections before birth and diagnose them after birth with the aim of stopping the infectious process [17, 22] Rubella is the most severe form of intrauterine infection. Rubella infection in the first trimester of pregnancy can expose the fetus to growth malformations such as hearing loss, heart malformation, visual impairment, and mental retardation [23, 24]. Fortunately, numerous seroepidemiological studies have led to generalized rubella vaccination, especially mothers in reproductive age, which will reduce the incidence of congenital rubella infection [25].

A large number of cerebral shunts are inserted annually in children admitted to the neurosurgery department for a variety of reasons $[17,18]$. Shunt complications are found in some cases that require emergency shunt placement [1921]. Considering the importance of this issue, this cross-sectional study was conducted to investigate complications of intraventriculopitonal shunt in children admitted to Rasool Akram hospital for two years (2013 -2016). The purpose of this study was to evaluate and compare complications and problems caused by cerebral shunt (based on imaging changes, clinical and laboratory findings) in children with congenital (intrauterine) infections, referred to as TORCH, with other types of shunts in children. 
Complications associated with shunts in children often lead to hospitalization and even death. By understanding shunt complications, determining causes of shunt insertion in children and diagnosis of complications caused by shunts, it would be possible to explain factors that lead to death or sequels and treat them effectively.

\section{MATERIAL AND METHOD}

In this descriptive, cross-sectional, case-control study, children with Ventriculo-peritoneal shunt were admitted to Rasoul-e-Akram hospital from the beginning of the year 2013 to the end of August, 2016. Sample size included 68 patients with ventriculo-peritoneal shunt admitted in Rasool Akram Hospital. From total 68 children, 47 patients were finally examined with VP shunt due to death in 13 patients (including 8 males and 5 females). The sampling was conducted as an easy method.

The data were recorded in checklists according to the variables.

Filling out the questionnaire for patients with VP shunt, determining the causes of non-infectious brain shunting, performing clinical examinations and determining other organs' involvement in patients with intrauterine infections were based on information that had been recorded in patients' files in the hospital.

data collection was done by blood sampling from patients, performing serological and general tests for determining the type of intrauterine infection, determining the type of maternal infection in children, identifying intrauterine and noninfectious causes, determining the time of shunt insertion in children with intrauterine infection and non-infectious group, determining disease course, mortality and peritonitis in both groups and finally, determining clinical response to surgical procedures in both groups.

Data were analyzed by SPSS software, version 24. Data were analyzed using central indexes such as mean and SD scattering indexes.

\section{RESULTS}

During this study, 68 children were admitted with a diagnosis of hydrocephalus. Totally, 13 children died during the time of the study.

Characteristics of expired patients included: $61.5 \%$ (8 cases) were male and $38.5 \%$ (5 persons) were girls with a mean age of $41.61 \pm 58.14$ days.

Characteristics of live (47) patients with VP shunt included: Of all 47 patients, 59\% (27patients) were male and 19 $(41 \%)$ were female with a mean age of $83+63$ days.

\subsection{Causes of Shunt Insertion}

The causes of shunt placement were infectious in $28 \%$ and noninfectious in resting patients. Characteristics of expired patients were compared to live patients in Table $\mathbf{1}$.

Table 1. Comparison between variables in alive and dead cases.

\begin{tabular}{|c|c|c|c|}
\hline $\begin{array}{c}\text { Duration of shunt insertion } \\
\text { (days) }\end{array}$ & $\begin{array}{c}\text { Male/female } \\
\text { Ratio }\end{array}$ & $\begin{array}{c}\text { Mean age } \\
\text { (month) }\end{array}$ & Variable \\
\hline $31.38+50.33$ & $8 / 5$ & $57.81+41.81$ & Dead cases \\
\hline $56.95+57.36$ & $27 / 19$ & $83.07+63.59$ & Alive cases \\
\hline
\end{tabular}

Causes of Shunt insertion in Patients who died during our study were Infectious in 4 patients. The other causes such as tumor, cerebral hemorrhage, and myelomeningocele were reported in other 9 patients. Congenital cytomegalovirus infection was reported in one patient. According to the results, $92 \%$ of expired patients had Shunt -induced meningitis and $7 \%$ shunt-induced peritonitis.

The characteristics of resting 47 patients with VP shunt included: Of all 47 patients, 59\% (27patients) were male and $19(41 \%)$ were female with a mean age of $83+63$ days.

Causes of shunt insertion were detected infectious in $28 \%$ and noninfectious in resting patients. Characteristics of expired patients were compared to live patients in Table $\mathbf{1}$. 


\subsection{The Course and Complications of Shunt Insertion}

Clinical symptoms of patients on admission: $33.3 \%$ patients had fever (38 degrees and higher), $50 \%$ nausea and vomiting, $50 \%$ headache, $16.7 \%$ seizure, $17.7 \%$ decreased level of consciousness and finally $25 \%$ with imbalance. Mechanical complications(Shunt obstruction and Shunt-induced cerebral hemorrhage) was reported in $8.2 \%(4$ cases).Infectious complications of cerebral palsy: Positive blood culture was found only in $8.5 \%$ patients. Peritonitis was detected in $10.9 \%$ (5 people). Lumbar puncture was performed only in $2.2 \%$ of patients. But, it was considered unnecessary in other cases. Meningitis was detected in $26.1 \%$ (12 cases) in the spinal fluid analysis. Resting $71.5 \%$ (33 cases) had no meningitis. Spinal fluid changes was observed in 12 patients, including leukocyte higher than 5 in $33.3 \%$ of patients and high level of protein in $25 \%$. Spinal fluid culture was obtained in $6.3 \%$.gender distribution was compared in both infectious and noninfectious groups by Fisher test in Table 2.

Table 2. Comparison between variables in cases with infectious and non-infectious causes of VP shunt insertion.

\begin{tabular}{|c|c|c|c|c|}
\hline $\begin{array}{c}\begin{array}{c}\text { Duration of shunt insertion } \\
\text { (days) } \\
P=0.8\end{array} \\
\end{array}$ & $\begin{array}{l}\text { Mechanical shunt complication } \\
\qquad \begin{array}{l}P=0.297\end{array}\end{array}$ & $\begin{array}{c}\text { Male/female } \\
\text { Ratio } \\
\mathbf{P}=1\end{array}$ & $\begin{array}{c}\text { Mean age } \\
\text { (month) } \\
P=0.7\end{array}$ & Variable \\
\hline $32.33+48.38$ & $2 / 12$ & $7 / 5$ & $78.83+80.63$ & Infectious causes \\
\hline $66.70+59.40$ & $2 / 32$ & $20 / 13$ & $85.38+58.34$ & Non Infectious causes \\
\hline
\end{tabular}

No difference was found in gender distribution $(\mathrm{P}$-value $=1.0)$. There was no significant difference in mean age between two groups with infectious and non-infectious origin $(\mathrm{P}$-value $=0.7)$. No considerable disparity was detected in the duration of shunt insertion between two groups $(\mathrm{P}-\mathrm{value}=0.8)$. Shunt-induced complications (mechanical complications such as shunt obstruction, cerebral hemorrhage) were measured by Fisher test. No difference was observed between two groups with infectious and noninfectious origin ( $\mathrm{P}-$ Value $=0.297$ ), but McNemar's test showed a clear difference in results (0.039). A meaningful difference was considered in shunt -induced mortality rate in both groups $(\mathrm{p}=0.0030)$. The risk of meningitis resulted from shunt insertion was expressively higher in group with infectious-induced shunt insertion $(\mathrm{P}$-value $=0.05)$. Risk of peritonitis was not considerably different between two groups with Fisher test (P-value=1.0).

\section{DISCUSSION}

The results of this study showed shunt complications in 68 patients with hydrocephalus. In total, 13 patients died due to shunt Complications and 47 survived. In a study by Deroquo et al. $29 \%$ of patients had a complication in the first year of shunt insertion. In this study, the greatest effect on complications occurrence was related to age distribution at the time of surgery [6]. Among patients who died in this study, 4 cases were suffering from hydrocephalus caused by congenital infections.For example, congenital cytomegalovirus infection was detected in one patient with VP shunt from birth time. In 9 other cases, causes of shunt insertion included tumor, cerebral hemorrhage, and myelomeningocele. Shunt infection was very common. The results showed that meningitis was reported in $92 \%$ of expired patients and peritonitis in $7 \%$. But the cause of death was due to underlying conditions, not cerebral shunt insertion. The previous study in this center was performed on 95 patients (56 males and 39 females) with a mean age of $27 \pm 5$ days of ventriculoperitoneal shunt insertion. Average age in the incidence of complications was $2.8 \pm 2.2$ years. Also, fracture and dislocation in cerebral shunt most often occurred after one year from the time of shunt insertion. Hematoma and bleeding were detected averagely after $22 \pm 25$ days. Infection occurred up to 6 months after shunt placement. The most common causes of complications were shunt occlusion (60\%) and infection (25.3\%). CT scan was able to show these complications in 58 cases $(62 \%)$, while $2.1 \%$ of CT scans did not demonstrate any changes versus previous ones. CT scan showed $50 \%$ and $87 \%$ sensitivity and specificity in detection of shunt obstruction, respectively . All cases of hemorrhage and hematoma were detectable on CT scan. However, CT scan had 20\% and $60 \%$ sensitivity and specificity in detection of shunt infection. In contrast, in cases of infectious shunts, CSF examination showed $92 \%$ hypoglycemia, $87.5 \%$ pleocytosis and $62.5 \%$ positive culture. CSF examination had $92 \%$ sensitivity, $82 \%$ specificity, positive predictive value of $63 \%$, and negative predictive value of $97 \%$, which considered this as an appropriate test for detection of infectious shunt cases. Clinical signs and symptoms were important in raising the diagnostic value of both tests. This raised the importance of performing lumbar fluid puncture, which unfortunately had been performed in small percentage of patients in current study.

In contrast to the current study, in a study by Karyatil et al. [7] two major shunt complications were abdominal pseudocyst and ascites. In this retrospective study, 15 patients were detected with abdominal pseudocyst caused in $60 \%$ cases with shunt functional complication. In 5 patients, ascites was found with abdominal symptoms [7]. In the clinical 
course of this study in survived patients, shunt mechanical complications were reported in only $8.7 \%$ (Shunt obstruction and shunt-induced cerebral hemorrhage). But, infectious shunt complications were discovered in a high percentage. In a study carried out by the Carpinen, the most common complications in shunt-affected individuals were obstruction and shunt infection. Shunt infection usually was realized as an unknown fever with undetermined origin (7). In our study, $33.3 \%$ had the clinical symptom of fever. Clinical manifestations in admitted patients matched more commonly with shunt infection. Fever was detected in $33.3 \%$, nausea and vomiting in 50\%, headache in $50 \%$, seizure in $16.7 \%$, decreased consciousness in $17.7 \%$ and imbalance in $25 \%$ of patients. In shunt infection, any type of neurological symptoms, even without fever, can be considered in favor of a shunt infection [1 - 4]. Unfortunately, lumbar puncture(which was absolutely necessary)was carried out in only $2.2 \%$ of patients in spite of presence of fever and neurological symptoms. Shunt meningitis was proven in 26\% (12 cases). Resting 97\% of patients received antibiotic due to fever and other symptoms without spinal fluid examination. Positive blood cultures were reported in $8.5 \%$ and positive spinal cord fluid culture in $6.3 \%$. $11 \%$ of patients had shunt-induced peritonitis. The current study, like other internal and external studies, showed that infectious complications of cerebral shunt were significantly common and might be the cause of death in patients [5 - 10]. Infection was the cause of Shunt dysfunction in $26 \%$ of survived patients in our study, which was several times more common compared to studies carried out in other countries(8-12\%) [16].

In the current study, in 47 live patients, congenital infection was the cause of shunt insertion in $28.3 \%$ of patients and the rest were due to non-infectious causes. However, there was no significant difference between the two groups regarding the course of the disease, age and other variables based on shunt insertion causes. But according to Jorabian et al. (Tehran), the most common cause of hydrocephalus and shunt placement in infants about 3 months of age was equiduct obstruction [15] with 3 deaths mortality and shunt complications including subdural effusion( 6 cases), shunt infection (4 cases) and shunt mechanical dysfunction( 8 cases) [15].

Radmannes et al. also reported shunt-induced infection in patients with myelomeningocele after shunt insertion [12].

The study of Behjati et al. on 97 premature infants with intracranial hemorrhage up to 6 months after birth in Tehran showed that shunt insertion was necessary in 35\%, mostly in patients with brain hemorrhage of grade 3 and higher. They also followed patients for around one year. They observed that from the total of 31 cases with brain shunt, cerebral shunt infection occurred in 11 cases [13]. Most of these infections occurred during the first 6 months of shunt placement often due to infection with normal skin flora during surgery, but some cases of infection reported several months after surgery probably related to the infection in a distant place (such as urinary tract infection). Other potential causes included skin injury at the place of shunt insertion or retrograde infection in distal catheter [15 - 17].

In a study by Di Rocco et al. [9], 773 patients were studied retrospectively, regarding the prevalence of shunt complications in the first year, their relationship with age, hydrocephalus etiology and type of shunt. Shunt Complications were reported in $29 \%$ of patients at the time of surgery had the greatest impact on the occurrence of shunt complications(9). In our study, the frequency of complications was relatively similar to these results. Shayanfar $e t$ al. results were similar to our current study [14]. In this study, 57 positive cerebrospinal fluid culture were detected including 30\% due to coagulase-negative staphylococci, $26 \%$ with Staphylococcus aureus, and 24.5\% with Klebsiella, Enterococcus and pseudomonas and other gram-negative bacteria. The most commonly used empirical antibiotics for the treatment of shunt infection (until determination of culture results) were recommended by combination of Vancomycin and Meropenem [14]. In a study by Dr. Pitetti [2], cases of suspected shunt dysfunction in emergency department showed that simple radiography was useful in evaluating shunt dysfunction in children, since some patients might refer with normal CT scans but abnormal radiological findings [2]. These results differed from that of Dr. Bruce [3]. In 296 patients with shunt complications, CT scans were superior to serial radiology [8]. In Mater [4], sensitivity and specificity of CT scan and serial radiography were so low that consultation with a neurosurgeon was proposed in patients with normal radiology and CT scan results [4]. We also used combination of imaging, clinical and laboratory findings to investigate shunt complications.

In a study by Carpinen [7], the most commonly occurring complication in patients with shunt insertion was obstruction and infection. Shunt infection usually presents itself as a febrile illness without localized sign [7]. Rocque $e t$ al. [8] suggested Shunt sampling as the best way to diagnose Shunt infection [8].

Ernell Kay studied 8 Swedish children with hydrocephalus and VP Shunt. Despite shunt obstruction, CSF examination could be normal. Although no case of infection was detected in that study, the CSF analysis was explained 
as a response to shunt obstruction [10]. In the current study, 33.3\% of patients had high leukocyte count in CSF analysis, $25 \%$ had high CSF protein and $8.8 \%$ of patients had positive CSF culture.

\section{CONCLUSION}

VP shunt Complications were reported in one-quarter of children, but mechanical shunt complications were uncommon in our study ( $8 \%$ of survived patients). The shunt mortality was relatively high (13 cases in total 68 patients) and more often in patients with non-infectious causes of shunt insertion, which was also due to meningitis. Shunt meningitis was considerably common in patients with an infectious cause of shunt insertion. Shunt infection in patients (meningitis, bacteremia and peritonitis) was relatively common and could result in death if not controlled. Antibiotics prescription without initial diagnostic measures (such as spinal fluid and ascites fluid examination and proper imaging) is not recommended because it will result in improper and incomplete treatments.

\section{ETHICS APPROVAL AND CONSENT TO PARTICIPATE}

Ethical Committee in the Pediatric Infectiuos Diseases (affiliates by Iran University of Medical Sciences) has reviewed and approved the Waiver of Authorization for use of protected health information (PHI) for research purposes for this study and the number is 92-01-131-25731.

\section{HUMAN AND ANIMAL RIGHTS}

No animals were used in this study. All human research procedures followed were in accordance with the ethical standards of the committee responsible for human experimentation (institutional and national), and with the Helsinki Declaration of 1975, as revised in 2008.

\section{CONSENT FOR PUBLICATION}

Consent for publication was obtained.

\section{CONFLICT OF INTEREST}

The authors declare no conflict of interest, financial or otherwise.

\section{ACKNOWLEDGEMENTS}

The research team wish to thank vice chancellor of research for their financial support and also children and their parents who contribute to this research.

\section{REFERENCES}

[1] Anderson CE, Garton HJL, Kestle JRW. Treatment of hydrocephalus with shunts.Principles and practice of pediatric neurosurgery. New York, NY: Thieme Medical Publishers 2007; pp. 109-30.

[2] Pitetti R. Emergency department evaluation of ventricular shunt malfunction: Is the shunt series really necessary? Pediatr Emerg Care 2007; 23(3): 137-41.

[http://dx.doi.org/10.1097/PEC.0b013e3180328c77] [PMID: 17413426]

[3] Lehnert BE, Rahbar H, Relyea-Chew A, Lewis DH, Richardson ML, Fink JR. Detection of ventricular shunt malfunction in the ED: Relative utility of radiography, CT, and nuclear imaging. Emerg Radiol 2011; 18(4): 299-305. [http://dx.doi.org/10.1007/s10140-011-0955-6] [PMID: 21523469]

[4] Mater A, Shroff M, Al-Farsi S, Drake J, Goldman RD. Test characteristics of neuroimaging in the emergency department evaluation of children for cerebrospinal fluid shunt malfunction. CJEM 2008; 10(2): 131-5. [http://dx.doi.org/10.1017/S1481803500009842] [PMID: 18371251]

[5] Arnell K, Olsen L. Distal catheter obstruction from non-infectious cause in ventriculo-peritoneal shunted children. Eur J Pediatr Surg 2004; 14(4): 245-9. [http://dx.doi.org/10.1055/s-2004-817896] [PMID: 15343464]

[6] Nishiyama K, Mori H, Tanaka R. Changes in cerebrospinal fluid hydrodynamics following endoscopic third ventriculostomy for shuntdependent noncommunicating hydrocephalus. J Neurosurg 2003; 98(5): 1027-31.

[7] Karppinen A. Complications of hydrocephalus shunting. Duodecim 2012; 128(16): 1691-8. [PMID: 23025153]

[8] Rocque BG, Lapsiwala S, Iskandar BJ. Ventricular shunt tap as a predictor of proximal shunt malfunction in children: A prospective study. J Neurosurg Pediatr 2008; 1(6): 439-43.

[http://dx.doi.org/10.3171/PED/2008/1/6/439] [PMID: 18518693] 
[9] O'Brien DF, Taylor M, Park TS, Ojemann JG. A critical analysis of 'normal' radionucleotide shuntograms in patients subsequently requiring surgery. Childs Nerv Syst 2003; 19(5-6): 337-41. [http://dx.doi.org/10.1007/s00381-003-0752-y] [PMID: 12740708]

[10] Kariyattil R, Steinbok P, Singhal A, Cochrane DD. Ascites and abdominal pseudocysts following ventriculoperitoneal shunt surgery: Variations of the same theme. J Neurosurg 2007; 106(5)(Suppl): 350-3. [PMID: 17566200]

[11] de Oliveira RS, Barbosa A, Vicente YA, Machado HR. An alternative approach for management of abdominal cerebrospinal fluid pseudocysts in children. Childs Nerv Syst 2007; 23(1): 85-90. [http://dx.doi.org/10.1007/s00381-006-0183-7] [PMID: 16944175]

[12] Radmanesh F, Nejat F. M El Khashab - Infection in myelomeningocele after VP shunt placementChilds Nervous System. Springer 2011.

[13] Behjati S, Emami-Naeini P, Nejat F. Incidence of hydrocephalus and the need to ventriculoperitoneal shunting in premature infants with intraventricular hemorrhage: Risk factors and outcome ... - Childs Nervous System. Springer 2011.

[14] Shayanfar N, Mohammadpour M. Invasive bacteria isolates from children with cerebral shunt and pattern of antimicrobial drug susceptibility in an Iranian referral pediatrics center Journal of Infectious ..., 2011 - SciELOBrasi

[15] Jouibari MF, Baradaran N, Amiri RS, Nejat F. Huge hydrocephalus: Definition, management, and complications Childs Nervous. Springer 2011.

[16] Noorbakhsh S, Farhadi M, Siadati A, et al. Study of Torch suspected infants. Iran J Pediat $2005 ; 15$ (Supp 1): 87.

[17] Marashi M, Mahmoodi M, Nategh R, et al. T Mokhtari-Azadprevalence of Rubella and Hemv antibodies among neonates with congenital defects in four provinces of Iran. Iranian J Virol 2011; 5(3)

[18] Sadighi J, Eftekhar H, Mohammad K. Congenital rubella syndrome in Iran. BMC Infect Dis 2005; 5: 44. [http://dx.doi.org/10.1186/1471-2334-5-44] [PMID: 15938744]

[19] Soleimanjahi H1. Fotouhi F1, Bamdad T1 evaluation of antibodies against Rubella virus in a mass campaign vaccination in Tehran, Iran. Indian J Virol 2010; 4(3\&4): 7-11.

[20] Siadati SA, Noorbakhsh S. CMV infection in primiparus pregnant women in Tehran. $6^{\text {th }}$ international con gress of pediatrics, $15-201994$, Tehran Iran.

[21] Noorbakhsh S, Memari F, Farhadi M, Tabatabaei A. Sensorineural hearing loss due to Toxoplasma gondii in children: A case-control study. Clin Otolaryngol 2008; 33(3): 269-73.

[http://dx.doi.org/10.1111/j.1749-4486.2008.01687.x] [PMID: 18559038]

[22] Noorbakhsh S, Siadati A, Farhadi M, et al. Role of cytomegalovirus in sensorineural hearing loss of children: A case-control study Tehran, Iran . Int J ped otolaryngo 2008; 72(2): 203-8.

[23] Esteghamati A, Gouya MM, Zahraei SM, Dadras MN, Rashidi A, Mahoney F. Progress in measles and rubella elimination in Iran. Pediatr Infect Dis J 2007; 26(12): 1137-41.

[http://dx.doi.org/10.1097/INF.0b013e3181462090] [PMID: 18043452]

[24] Zahraeia SM, Marandi A, Sadrizadehc B, et al. Role of National Immunization Technical Advisory Group on improvement of immunization programmes in the Islamic Republic of Iran Vaccine 2010; 28(suppl 1): A35-8.

[25] Doroudchi M, Dehaghani AS, Emad K, Ghaderi AA. Seroepidemiological survey of rubella immunity among three populations in Shiraz, Islamic Republic of Iran. East Mediterr Health J 2001; 7(1-2): 128-38.

[PMID: 12596962]

(C) 2018 Shahi et al.

This is an open access article distributed under the terms of the Creative Commons Attribution 4.0 International Public License (CC-BY 4.0), a copy of which is available at: https://creativecommons.org/licenses/by/4.0/legalcode. This license permits unrestricted use, distribution, and reproduction in any medium, provided the original author and source are credited. 